\title{
Global anisotropy of arrival directions of ultra-high-energy cosmic rays: capabilities of space-based detectors
}

\author{
O. E. Kalashev ${ }^{1}$, B. A. Khrenov², P. Klimov², S. Sharakin ${ }^{2}$ and \\ S. V. Troitsky ${ }^{1}$ \\ ${ }^{1}$ Institute for Nuclear Research of the Russian Academy of Sciences, \\ 60th October Anniversary Prospect 7a, 117312, Moscow, Russia \\ ${ }^{2}$ D.V. Skobeltsin Institute of Nuclear Physics, \\ M.V. Lomonosov Moscow State University, Moscow 119992, Russia \\ E-mail: st@ms2.inr.ac.ru
}

\begin{abstract}
Planned space-based ultra-high-energy cosmic-ray detectors (TUS, JEMEUSO and S-EUSO) are best suited for searches of global anisotropies in the distribution of arrival directions of cosmic-ray particles because they will be able to observe the full sky with a single instrument. We calculate quantitatively the strength of anisotropies associated with two models of the origin of the highest-energy particles: the extragalactic model (sources follow the distribution of galaxies in the Universe) and the superheavy dark-matter model (sources follow the distribution of dark matter in the Galactic halo). Based on the expected exposure of the experiments, we estimate the optimal strategy for efficient search of these effects.
\end{abstract}

PACS number: 98.70.Sa 


\section{Introduction}

The next step in the studies ultra-high-energy (UHE) cosmic rays (CRs) is related to the use of space-based detectors observing the fluorescent light induced by air showers in the terrestrial atmosphere with large exposures. It is expected that these instruments will help us to shed light on the origin of the highest-energy cosmic particles which remains unknown up to now.

One of the important signatures of particular UHECR models is the global anisotropy of arrival directions of the highest-energy events. For instance, models where the origin of UHECRs is attributed to the acceleration in astrophysical objects (so-called "bottom-up" scenarios) naturally predict that the distribution of arrival directions follows the distribution of these cosmic accelerators. In the most common scenario with extragalactic protons and/or nuclei, the patterns of the distribution of galaxies in the nearby Universe should be seen [1] on the UHECR skymap because of the limited propagation length of these particles due to the GZK effect [2] or nuclear photodisintegration. Strong suppression of the cosmic-ray flux is predicted at highest energies in these models. On the other hand, the "top-down" models [3] (see Refs. [4] for reviews and references) with the distribution of sources in the Galactic halo following that of the dark matter (which is the case for the superheavy dark-matter (SHDM) particles and some of topological defects) predict [5] the Galactic center-anticenter asymmetry due to the non-central position of the Sun in the Galaxy (see Refs. [6, 17, 8] for extensive discussions). Models of this kind predict continuation of the cosmic-ray spectrum and gamma-ray dominance beyond $10^{20} \mathrm{eV}$.

Currently, neither the spectrum nor anisotropy observations can definitely favour one of the two scenarios at the highest energies, $E \sim 10^{20} \mathrm{eV}$. Indeed, the AGASA experiment claims [9] the super-GZK continuation of the spectrum while the HiRes collaboration reports [10] the observation of the Greisen-Zatsepin-Kuzmin (GZK) cutoff (data of other experiments, including recent results of the Pierre Auger Observatory (PAO) [11, 12, are not yet conclusive: though the unsuppressed continuation of the spectrum is excluded by the Auger data, the cutoff in the spectrum is not clearly seen). The limits on the gamma-ray fraction in the primary cosmic-ray flux (currently the most restrictive ones arise from the AGASA and Yakutsk muon data at $E>10^{20} \mathrm{eV}$ [13], from the Yakutsk muon data at $E>4 \cdot 10^{19} \mathrm{eV}[14$ ] and from the Pierre Auger Observatory data on the shower geometry at $E>2 \cdot 10^{19} \mathrm{eV}$ and $E>10^{19} \mathrm{eV}$ [15]) disfavour the SHDM scenario and even exclude it for particular values of the dark-matter parameters (see e.g. Ref. [16] for a more detailed discussion of some of these limits). Current experiments do not report any significant deviations from the global isotropy at the highest energiest. This is however not conclusive both due to the low statistics and due to a limited field of view of any terrestrial-based installation.

The steeply falling spectrum of cosmic rays makes it very difficult to obtain a reliable

$\ddagger$ After this paper was submitted, PAO reported a significant deviation from isotropy [17]. The definite interpretation of this effect awaits further data (see e.g. Ref. [18] for discussion). 
measure of the global anisotropy in any combination of terrestrial experiments. Indeed, the relative systematic difference in the energy estimation between two installations located in different parts of the Earth (and thus observing different parts of the sky) can hardly be made smaller than some $\sim 15 \%$. Such a relative error would give $\sim 30 \%$ higher integral flux seen by one of the experiments with respect to the other at the same reconstructed energy. Thus, possible observations of global anisotropy can be attributed both to a physical effect and to unknown systematics in the energy determination. Moreover, a seemingly isotropic distribution of the arrival directions over the sky might represent a physically anisotropic one masked by the systematic effects.

On the other hand, the planned space-based experiments, e.g. TUS [19], JEMEUSO [20] or S-EUSO [21], will provide a unique opportunity to observe full sky with a single detector. While not being free from systematic uncertainties in the energy determination, an experiment of this kind would not introduce strong directiondependent systematics and thus would be able to perform the studies of the global anisotropy at high confidence.

One may expect that in future space-based experiments with their huge exposures, particular sources of UHECR will be determined on event-by-event basis for the case of the astrophysical scenario. This is not an easy task, however: limited angular resolution together with large numbers of events would lead to identification problems similar to thoose of the gamma-ray astronomy\& but strongly enhanced due to magnetic deflections of the charged cosmic-ray particles. The searches for global patterns in the distribution of UHECR arrival directions will thus be important in any case.

A robust method for the study of any global asymmetry in the arrival directions is the harmonic analysis (see e.g. Ref. [23]). It works perfectly if the predicted effect may be clearly seen in the first few harmonics but requires large statistics to reveal/exclude more complicated patterns. Here, we determine the optimal strategy for the searches of global anisotropies even with the low-resolution experiments (TUS) and for short exposure times.

The strategy is to fix two regions of the sky (not necessary covering full $4 \pi$ ) which are expected to provide the strongest contrast in over/underdensity of events with respect to the null hypothesis of the isotropic distribution. The shape and the size of these regions, as well as the energy range of the events, are determined a priory in order to balance the strength of the expected anisotropy (increasing for smaller regions and higher energies) and its statistical significance. The aim of this paper is to simulate optimal regions and energies for TUS, JEM-EUSO and S-EUSO, suitable to test the scenarios of extragalactic sources and of decays of superheavy dark matter concentrated in the Galactic halo. To this end, we perform new and improved (with respect to previous works) simulations of the distribution of arrival directions expected in both cases.

$\S$ See e.g. Refs. 22 for discussions of statistical methods of analysis of photon-by-photon EGRET data. Currently, the gamma-ray sky at energies $(\sim 0.1 \div 1) \mathrm{GeV}$ contains 101 identified source, 170 unidentified ones and a strong non-uniform diffuse background of unidentified origin. 
The paper is organised as follows. In Sec. 2 and Sec. 3, we discuss our simulations for extragalactic sources and for SHDM decays, respectively. Sec. 4 gives specific predictions for three coming spaceborn experiments, TUS, JEM-EUSO and S-EUSO. Sec. 5 contains our brief conclusions. Some technical details are collected in Appendix A.

\section{The "bottom-up" scenario: sources follow the distribution of extragalactic luminuous matter}

A number of astrophysical sources were suggested where acceleration of cosmic rays up to the highest energies can take place (see Refs. 24] for reviews and summary). A common assumption is that the distribution of the sources follows that of luminuous matter, that is of galaxies. We should stress that this approach does not imply that all galaxies emit cosmic rays of UHE energies - this is certainly ruled out; instead, it assumes that the number density of the sources is, on average, proportional to the number density of the galaxies. This latter assumption is true for most of the suggested cosmic accelerators (active galactic nuclei, starburst galaxies, gamma-ray bursts etc.) Our approach assumes that the number of sources within the GZK sphere is large enough $\left(\gtrsim 10^{2}\right)$ so that the large-scale structure of the Universe traces well their distribution within $\sim 100 \mathrm{Mpc}$. The assumption does not work for the case of only few sources and very strong intergalactic magnetic fields (see Sec. 2.3).

Interaction of UHE hadrons with cosmic background radiation limits the propagation distance at high energies; hence, a limited part of the Universe may contain the sources of UHECRs detected at the Earth. Non-uniform distribution of matter in this part should reflect itself in the distribution of the arrival directions of cosmic rays [1]. For this study, the following scheme was used.

- We calculated the density function of the sources $n(l, b, d)$ as the number density of galaxies for a given direction (Galactic coordinates $l, b$ ) and distance $d$.

- By making use of a numerical propagation code [25], we estimated the fraction $f\left(d, E_{\min }\right)$ of surviving hadrons with $E>E_{\min }$ at the distance $d$ from the source, assuming either proton or iron injected primaries.

- The density of the sources $n(l, b, d)$ was convolved with the survival function $f(d)$ to obtain the expected distribution of the UHECR arrival directions.

\subsection{The density function of the sources}

Most of the previous studies [1] used the PSCz catalog [26] to reconstruct the source density. However, due to a limited angular resolution of the IRAS instrument used to compile the catalog it might not resolve all galaxies in the regions of high density (clusters) [27] and thus may systematically diminish the expected anisotropy due to underestimation of high number densities of galaxies. For now, the most complete full-sky catalog of galaxies is the 2MASS XSC [28]. Redshifts are known only for a small fraction of the 2MASS galaxies; the photometric redshifts are proven to be 
a good measure of the distance to the rest of them [29]. However, the precision of photometric redshifts is insufficient at low distances; moreover, artificial nearby sources may appear due to uncertainties in the determination of the Galactic extinction. We use another full-sky galaxy catalog, LEDA [30], to identify the density function at $d<30 \mathrm{Mpc}$. A volume-limited sample of LEDA [31] for $d<50 \mathrm{Mpc}$ is used, and the slice $30 \mathrm{Mpc}<d<50 \mathrm{Mpc}$ is used for calibration of the density of the sources (in arbitrary units) determined from LEDA and from 2MASS XSC photometric redshifts. The latters are calculated following Ref. [32], using the Galactic extinction of Ref. [33], and are used for $d>30 \mathrm{Mpc}$. The details of calculation are given in Appendix A. Our sample is complete up to the distances of order $270 \mathrm{Mpc}$ (we assume $H_{0}=73 \mathrm{~km} / \mathrm{s} / \mathrm{Mpc}$ ).

The actual density of the sources is much less than that of all galaxies. This means that the density function should be smoothed on a reasonable scale to suppress unphysical fluctuations. For this study, we smooth the function $n(l, b, d)$ at the scale of $10 \mathrm{Mpc}$ in $d$ and $\sim 5^{\circ}$ on the celestial sphere. The latter value is also motivated by the uncertainty due to deflection of charged hadrons in the cosmic magnetic fields (see Sec. 2.3 for discussion) and by the experimental angular resolution.

\subsection{Calculation of the fraction of surviving hadrons}

We use a detailed propagation code [25] which is based on kinematic equations written in the expanding Universe and accounts for numerous interaction processes, tracing the propagation of nuclei (iron and lighter), nucleons, gamma-rays, electrons and neutrinos. Parameters of the simulation have been chosen [34] in such a way as to provide the best fit to the HiRes data [10]: for injected protons, the assumed spectral index at the source is $\alpha=2.55$ and the maximal injected energy is $E_{\max }=1.3 \cdot 10^{21} \mathrm{eV}$; for iron these parameters are $\alpha=2.2$ and $E_{\max }=1.7 \cdot 10^{22} \mathrm{eV}$, correspondingly. In both cases, the best fit corresponds to the absence of the source evolution and to the zero distance to the nearest source. The same parameters provide reasonable fits to the data of the Pierre Auger surface detector [11] if the energy calibration independent of the fluorescent yield [12] is used; see Ref. [35] for other fits to the Auger spectrum.

The functions $f\left(d, E_{\min }\right)$ are plotted in Figs. 1, 2 for different injected primaries and $E_{\text {min }}$. Since our sample is complete up to $d \sim 270 \mathrm{Mpc}$, we see (cf. Fig. 1, 2) that the study makes sence for $E \gtrsim 7 \cdot 10^{19} \mathrm{eV}$.

These results are compared to a related study [36] in Figs. 3, 4. The agreement between the two simulations is reasonably good though ours predict slightly larger contribution from distant sources.

\subsection{Effect of the cosmic magnetic fields}

The previous discussion did not take into account the effect of the cosmic magnetic fields on the propagation of charged cosmic particles. This effect is determined by bending of particle trajectories and is therefore twofold: firstly, the trajectories become

longer and secondly, they do not point back to the origin. The first effect may have 


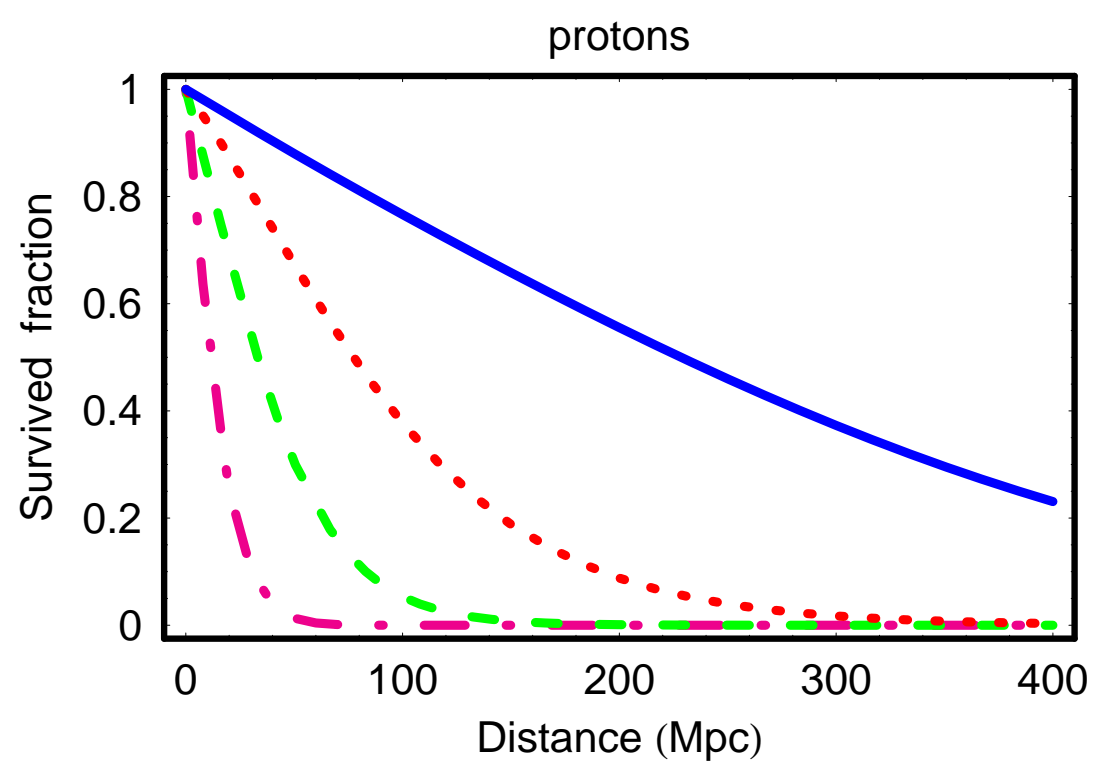

Figure 1. The fraction of surviving hadrons with $E>E_{\min }$ as a function of the distance $d$ from the proton-emitting source with spectral index $\alpha=2.55$ and a cutoff at $1.3 \cdot 10^{21} \mathrm{eV}$. Solid line, $E_{\min }=4 \cdot 10^{19} \mathrm{eV}$; dotted line, $E_{\min }=7 \cdot 10^{19} \mathrm{eV}$; dashed line, $E_{\min }=10^{20} \mathrm{eV}$; dash-dotted line, $E_{\min }=2 \cdot 10^{20} \mathrm{eV}$.

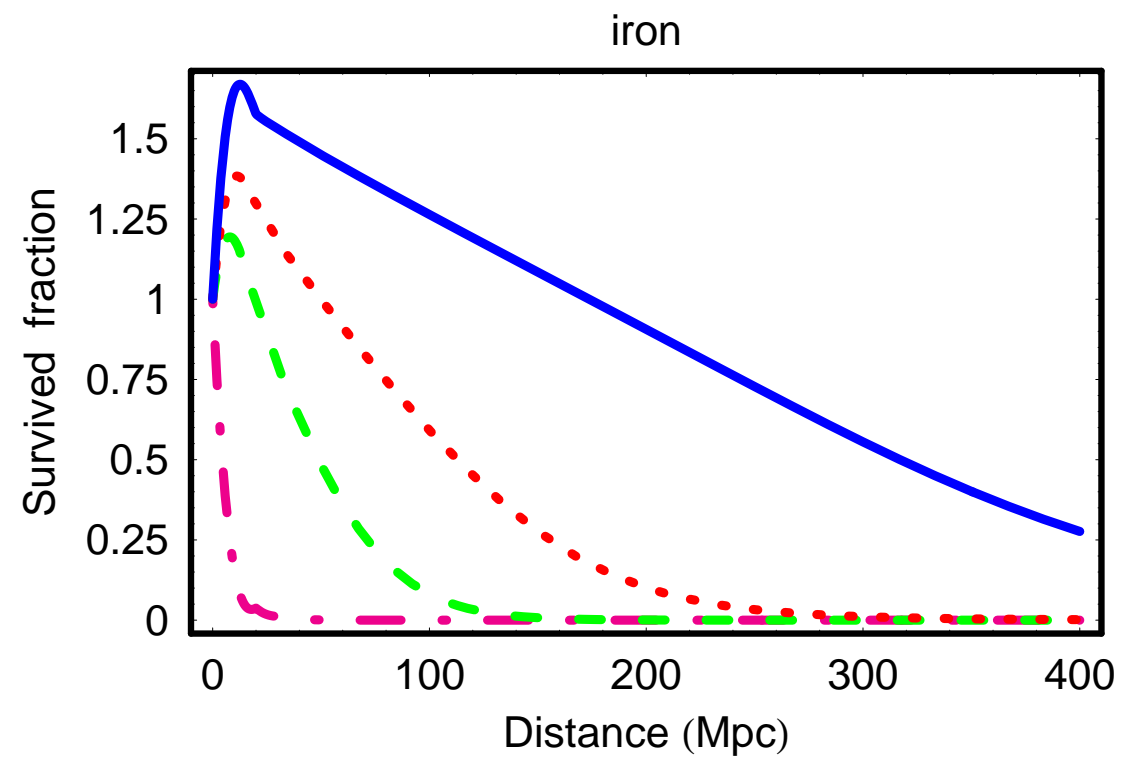

Figure 2. The fraction of surviving hadrons with $E>E_{\min }$ as a function of the distance $d$ from the iron-emitting source with spectral index $\alpha=2.2$ and a cutoff at $1.7 \cdot 10^{22} \mathrm{eV}$. Solid line, $E_{\min }=4 \cdot 10^{19} \mathrm{eV}$; dotted line, $E_{\min }=7 \cdot 10^{19} \mathrm{eV}$; dashed line, $E_{\min }=10^{20} \mathrm{eV}$; dash-dotted line, $E_{\min }=2 \cdot 10^{20} \mathrm{eV}$. Note that the number of particles is first enhanced at dosens Megaparsecs from the source because of prompt disintegration of heavy nuclei into a number of light ones and only then becomes suppressed due to the GZK effect. 




Figure 3. Horizons of protons: proton-injecting sources at distances larger than $D$ contribute a fraction $k$ of the observed flux. Solid lines, $k=0.3$; dashed lines, $k=0.1$. Black lines, this study; gray lines, Ref. [36. Injection spectral index $\alpha=2.7$.



Figure 4. Same as Fig. 3 but for iron nuclei injected with the spectral index $\alpha=2.2$. 
important consequences for the spectrum if the change in the trajectory length becomes comparable to the attenuation length of the particle; the second effect may change the expected skymap significantly.

For our purposes, it would be useful to separate the effects of the extragalactic magnetic fields from those of the magnetic field of the Milky Way.

2.3.1. Extragalactic magnetic fields. Direct information about the strength of intergalactic magnetic fields is not available and the corresponding estimates vary by orders of magnitude. Magnetic fields of order $10^{-11} \mathrm{G}$ in the most part of the local Universe (except dense clusters of galaxies) are predicted by simulations of Ref. [37] while fields as strong as $10^{-8} \mathrm{G}$ in voids are advocated in Ref. [38. Detailed calculations of cosmic-ray trajectories were given e.g. in Refs. [37, 38, for both cases. A typical deflection angle $\chi$ of a particle with energy $E$ and charge $Z$ may be roughly estimated as

$$
\chi \lesssim 0.1^{\circ} \frac{7 \cdot 10^{19} \mathrm{eV}}{E} \frac{B}{10^{-11} G} Z
$$

where $B$ is the typical magnetic-field strength in the voids. We see that for the strongfield scenario $\left(B \sim 10^{-8} \mathrm{G}\right)$, cosmic-ray trajectories are bend strongly and become entangled $\left(\chi \sim 360^{\circ}\right)$ even for protons $(Z=1)$ of $E \sim 7 \cdot 10^{19} \mathrm{eV}$ (in this case, even a few sources within $\sim 100 \mathrm{Mpc}$ would not result in significant anisotropy of arrival directions). For less extreme values of $B \lesssim 10^{-9} \mathrm{G}$, typical deflections of protons do not exceed a few degrees at the super-GZK energies so that the analysis of this work remains applicable. For the weak-field scenario of Ref. [37] which we assume in what follows, deflections of nuclei as heavy as iron are of order of, or less than the experimental angular resolution at $E \gtrsim 7 \cdot 10^{19} \mathrm{eV}$.

The effect of the lengthening of trajectories on the spectrum of various nuclei was studied in detail in Ref. [39]; it may be neglected within our precision for $B<10^{-9} \mathrm{G}$ and the energies of interest (see e.g. Fig. 10 of Ref. [39]).

2.3.2. Galactic magnetic fields. Much stronger $\left(\sim 10^{-6} \mathrm{G}\right)$ and somewhat better studied magnetic fields are present in the Milky Way (see e.g. Refs. [40] for reviews of various models with applications to UHECR deflections). However, their strength is partially compensated by the relatively small size they occupy (strong fields are localized within the Galactic disk and possibly in the central regions of the bulge). At $E \gtrsim 7 \cdot 10^{19} \mathrm{eV}$, the deflections are always small $\left(\sim 1^{\circ}\right)$ for protons but may be important for heavier nuclei, at least in the directions along the Galactic plane. The actual skymap at low Galactic latitudes $b$ depends strongly on the particular model of the magneticfield structure; however at relatively high $|b| \gtrsim 50^{\circ}$ the deflections are always small and the pattern of cosmic structures at these latitudes (where e.g. the Virgo supercluster is located) remains unchanged. The lengthening of particle trajectories (except possibly for the rare ones which hit the central region of the Galaxy and may get entangled) is negligible as compared to the attenuation length. 
To summarize this section, the results of our study are not significantly affected by the account of intergalactic magnetic fields of $B \lesssim 10^{-9} \mathrm{G}$ for protons and $B \lesssim 10^{-10} \mathrm{G}$ for heavier nuclei and by the account of the Galactic magnetic fields - everywhere for protons and at high Galactic latitudes, $|b| \gtrsim 50^{\circ}$, for heavy nuclei. The actual account of these magnetic fields is strongly model-dependent and is beyond the scope of this paper.

\section{The "top-down" scenario: sources follow the distribution of the Galactic dark matter}

In "top-down" models, UHECRs originate from decays of superheavy particles (the latters themselves may be produced in decays or collisions of the topological defects). The distribution of sources thus follows the distribution of the initial particles; for many realistic models it corresponds to the distribution of the dark matter. Decays of particles from the Milky-Way halo dominate the cosmic-ray flux at high energies in this case. At lower energies, this flux should be supplemented by a contribution of astrophysical sources to explain the observed spectrum. Due to a non-central position of the Sun in the Galaxy, the flux should be higher from the direction of the Galactic center than from the opposite one.

Decays of the SHDM particles may be described in a more or less model-independent way because the most important physical phenomenon of relevance is hadronization which involves light particles and is well understood. Denote $x \equiv \frac{2 E}{M_{X}}$, where $E$ is the energy of a decay product of the SHDM particle with mass $M_{X}$. Then for $10^{-4} \lesssim x \lesssim 0.1$, spectra of the decay products calculated by various methods [7, 41] are in a good agreement with each other; moreover, the shape of the spectral curve $\frac{d N}{d E}(x)$ does not depend on $M_{X}$ [7]. For this study, we use the spectra of decay products from Ref. [7] Ш]

The SHDM decay rate is determined by the concentration $n_{X}$ and lifetime $\tau_{X}$ of the SHDM particles, $n_{X}=n_{X} / \tau_{X}$. The flux of secondary particles at the Earth is then determined by

$$
j=\mathcal{N} \frac{1}{\tau_{X}} \frac{d N}{d E}
$$

where

$$
\mathcal{N}=\int d^{3} r \frac{n_{X}(\mathbf{r})}{4 \pi r^{2}}
$$

is the geometrical factor ( $\mathbf{r}$ is the radius-vector from the Earth; though in principle one should integrate over the Universe and account for relativity effects, in most interesting cases the dominant contribution comes from the Galactic halo [5]). The mass $M_{X}$ is subject to cosmological limits (see e.g. Ref. [42] and references therein); the lifetime $\tau_{X}$ is much less restricted.

\footnotetext{
\| We thank M. Kachelrieß for providing numerical tables of the functions caculated there.
} 
Since the decays of SHDM particles may describe only the highest-energy part of the spectrum, the flux is assumed to be a sum of two components, one of which, $F_{\mathrm{EG}}$, corresponds to the "bottom-up" contribution (we use the results of Sec. 2 to model it) while the second one, $F_{\mathrm{DM}}$, is due to SHDM decays. Neglecting the contribution of SHDM outside the Milky-Way halo, one may rewrite the integral in Eq. (1) in the variables related to the Galaxy, namely the distance $R$ to the Galactic center and the angle $\theta$ between the directions "observer - Galactic center" and "observer - integration point". This is useful because the distribution of the dark matter in the Galaxy depends on $R$. Though the actual shape of the distribution is currently a subject of debates, the profile

$$
n(R)=\frac{n_{0}}{\left(\frac{R}{R_{S}}\right)^{\alpha}\left(1+\frac{R}{R_{S}}\right)^{3-\alpha}},
$$

is often used, where $R_{S}=45 \mathrm{kpc}$ and $\alpha=1$ for a popular NFW model [43]. We take the size of the halo $R_{h}=100 \mathrm{kpc}$ and the distance from the Sun to the Galactic center $r_{\odot}=8.5 \mathrm{kpc}$ to obtain

$$
\int d^{3} R n_{X}(r)= \begin{cases}2 I_{1}+I_{2}, & 0 \leq \theta \leq \pi / 2 \\ I_{2}, & \pi / 2 \leq \theta \leq \pi\end{cases}
$$

where

$$
I_{1}=\int_{r_{\odot} \sin \theta}^{r_{\odot}} n_{1}(R) d R, \quad I_{2}=\int_{r_{\odot}}^{R_{h}} n_{1}(R) d R, \quad n_{1}(R)=\frac{n(R)}{\sqrt{R^{2}-r_{\odot}^{2} \sin ^{2} \theta}}
$$

(note that the corresponding equation of Ref. [8] works only for $0 \leq \theta \leq \pi / 2$ ).

Each particular experiment has its own direction-dependent exposure $A(l, b)$, so the observed flux is a convolution of $F_{\mathrm{EG}}+F_{\mathrm{DM}}$ with $A$. For instance, AGASA sees the Northern sky and has higher exposure towards the Galactic anticenter while Southern experiments (SUGAR and PAO) have higher exposures towards the Galactic center. To simulate the expected distribution of events, we fit the observed AGASA spectrum with the sum of $F_{\mathrm{EG}}$ and $F_{\mathrm{DM}}$ and find the $95 \%$ CL regions for the three parameters: the coefficients at $F_{\mathrm{EG}}$ and $F_{\mathrm{DM}}$ (the latter is related to $\tau_{X}$ ) and $M_{X}$. We impose additional constraints on the parameters. First, $M_{X}$ should satisfy the bounds of Ref. [42] and should be at least twenty times larger than the energy corresponding to the last point in the spectrum being fitted (in order to always be in a safe region $x \lesssim 0.1$ ); these bounds are not very restrictive. However, we impose the limits on the gamma-ray fraction as reported in Refs. [13, 14, 15] which exclude a significant part of the parameter space. Details of the fitting will be reported elsewhere; here we will present the results for two particular fits: the best fit for the AGASA spectrum (which is marginally consistent with gamma limits) and the fit with the minimal contribution from SHDM (consistent with gamma limits automatically) still allowed within 95\% CL region of parameters. We note that the results for the best fit of the PAO surface-detector spectrum [11] (calibrated as suggested in Ref. [12]) lay between these two. 


\section{Predictions for space-based experiments}

\subsection{Experiments}

The space detector TUS [19] is under construction and is planned to be launched in 2010. The TUS lower energy threshold is estimated as $7 \cdot 10^{19} \mathrm{eV}$ (with almost energyindependent sensitivity at higher energies). The UHECR arrival direction accuracy is different for different zenith angles $\theta$ : for $\theta<30^{\circ}$, the direction is estimated only roughly as being vertical in the error cone of $30^{\circ}$; for $30^{\circ} \lesssim \theta \lesssim 60^{\circ}$ the error approaches $10^{\circ}$ and for $60^{\circ} \lesssim \theta \leq 90^{\circ}$ the error is less than $10^{\circ}$. We used the events with $30^{\circ} \lesssim \theta \lesssim 90^{\circ}$ in our estimates; the exposure factor per one year of operation is about $3000 \mathrm{~km}^{2} \mathrm{sr}$ for these cuts. The TUS direction-dependent exposure $A(l, b)$ is determined by the satellite orbit parameters and by the zenith angle cut. It can be conveniently parametrized by a sum of a monopole and a quadrupole over the celestial sphere.

The JEM-EUSO detector [20] (to be installed in 2012 on board of the International Space Station, ISS) is expected to have an instantaneous aperture of $\sim 6 \cdot 10^{5} \mathrm{~km}^{2} \mathrm{sr}$ and a duty cicle of $\sim 20 \%$. JEM-EUSO will be sensitive to cosmic rays with energies $E \gtrsim 4 \cdot 10^{19} \mathrm{eV}$; the energy-dependent sensitivity is reported in Ref. [20]. The angular resolution of the detector is a few degrees; the ISS orbit parameters result in an almost uniform exposure over the sky (we therefore assume $A(l, b)=$ const for JEM-EUSO).

S-EUSO [21] is a proposed detector with an instantaneous aperture of $\sim 2$. $10^{6} \mathrm{~km}^{2}$ sr. The proposal has been submitted to the ESA "Cosmic Vision" program; the proposed launch date is 2017 . For S-EUSO we assume the duty cycle, energy-dependent sensitivity and $A(l, b)$ similar to those simulated for JEM-EUSO.

\subsection{Large-scale observables and optimisation}

To test one of the hypotheses discussed in Sec. 2, 3, we divide the sky into three parts: part 1 corresponds to the strongest expected excess of events over the uniform distribution, part 2 corresponds to the strongest deficit and part 3 is the rest of the sky. The shape and size of the parts, as well as the energy range, are determined from a priory simulations in order to maximize the signal. The observable of relevance is the difference between numbers of events in regions 1 and $2, n_{1}-n_{2} \equiv \Delta$. Though this strategy is best suited for TUS with its relatively low aperture and precision, it will be useful for analysis of early JEM-EUSO and S-EUSO data as well, especially at the highest energies.

Let $p_{i}, i=1,2,3$, be the probability of an event to arrive from the $i$ th region, $p_{1}+p_{2}+p_{3}=1$; let $N$ be the total number of events in the sample. Then the probability to have $n_{1}$ events in the region 1 and $n_{2}$ events in the region 2 is given by the multinomial distribution,

$$
P_{N}\left(n_{1}, n_{2}\right)=\frac{N !}{n_{1} ! n_{2} !\left(N-n_{1}-n_{2}\right) !} p_{1}^{n_{1}} p_{2}^{n_{2}} p_{3}^{N-n_{1}-n_{2}} .
$$




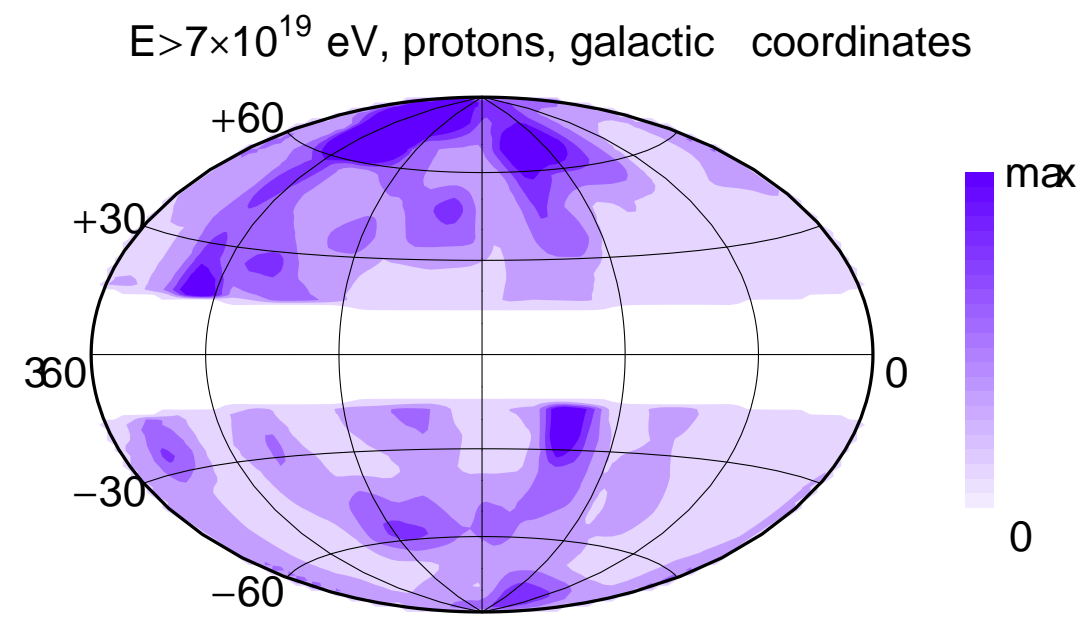

Figure 5. Expected density of events with $E>7 \cdot 10^{19} \mathrm{eV}$ from proton-emitting extragalactic sources plotted on a skymap in galactic coordinates for uniform exposure. Darker regions correspond to larger density (linear scale). The white band corresponds to the zone of avoidance $\left(|b|<15^{\circ}\right)$ cut out of the sample. Numerical values of the expected densities of events for this and other cases are available online from http://livni.inr.ac.ru/UHECRskymaps/.

The probability to have $n_{1}-n_{2}=\Delta$ is

$$
\begin{aligned}
P(\Delta)= & \left(1-p_{1}-p_{2}\right)^{N+\Delta} p_{2}^{-\Delta} \frac{\Gamma(N+1)}{\Gamma(N+\Delta+1) \Gamma(1-\Delta)} \\
& \times{ }_{2} F_{1}\left(-\frac{N+\Delta}{2},-\frac{N+\Delta-1}{2}, 1-\Delta, \frac{4 p_{1} p_{2}}{\left(1-p_{1}-p_{2}\right)^{2}}\right),
\end{aligned}
$$

where $\Gamma$ and ${ }_{2} F_{1}$ are the Gamma function and the hypergeometric function, respectively; $-N \leq \Delta \leq N$.

For each particular choice of the regions 1 and 2 and of the minimal energy, we calculate $P_{N}(\Delta)$ as a function of $N$ for the cases of the expected signal and of the null hypothesis of isotropic cosmic-ray flux (see Ref. [44] for a similar study). We expect that an experiment is able to confirm/exclude the hypothesis at the confidence level $\eta$ if

$$
P_{N}\left(\Delta_{\text {signal }}-\Delta_{\text {null }} \geq 0\right)>1-\eta .
$$

For a given $\eta$, we determine the required number of events $N$. Minimization of the time required to collect this number of events (estimated in each case using the spectrum relevant for the model we test) results in the choice of the optimal regions and energy.

\subsection{Results for the "bottom-up" scenario}

Figure 5 presents the expected density of events for uniform exposure simulated under conditions described in Sec. 2. The strongest excess of events is expected along the Supergalactic plane, in particular from the Virgo cluster and the Perseus-Pisces Supercluster. Compared to the previous studies, ours suggests a stronger contrast 


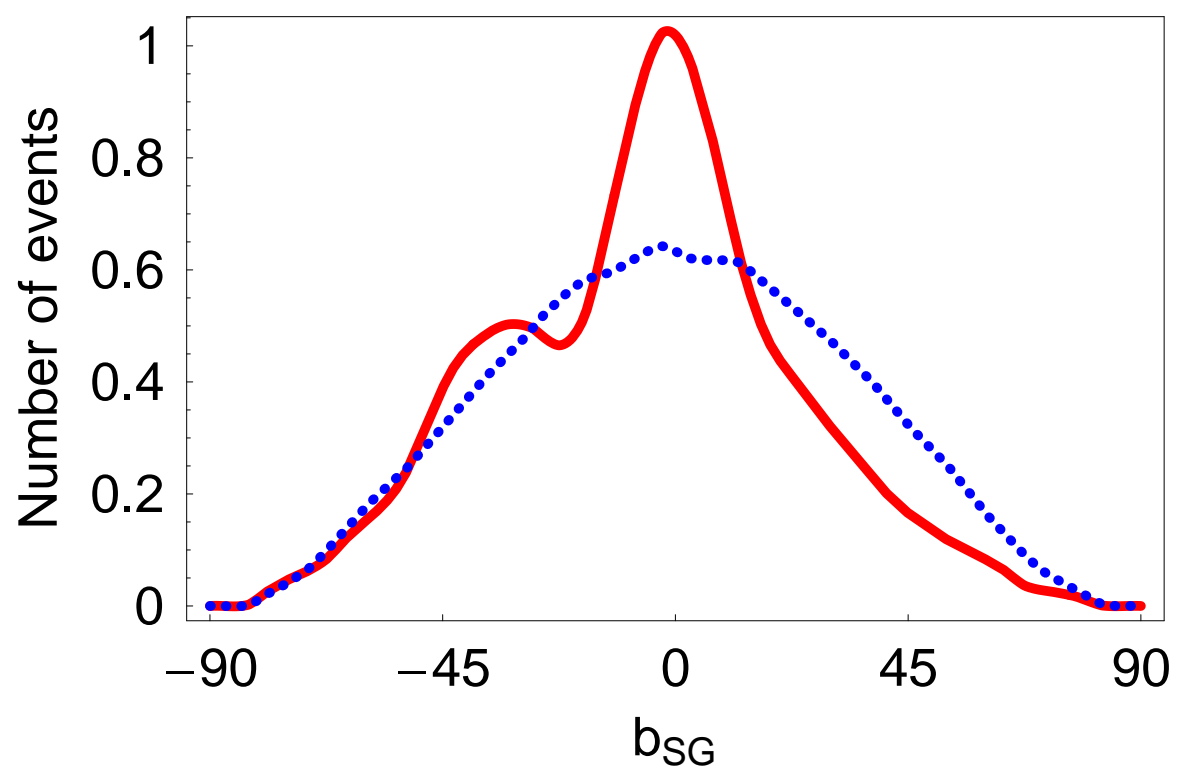

Figure 6. Expected distribution of events (arbitrary units) with $E>7 \cdot 10^{19} \mathrm{eV}$ from proton-emitting extragalactic sources (thick line) in the supergalactic latitude $b_{\mathrm{SG}}$, assuming TUS exposure. The dashed line corresponds to the isotropic distribution.

between local voids and filaments, probably due to the contrast in the source density function (cf. the IRAS-2MASS difference discussed in Sec. 2.1).

To determine the optimal choice of regions 1 and 2, we tested various cuts in galactic and supergalactic coordinates and their combinations. Not surprisingly, the strongest contrast is achieved in distribution of events in the supergalactic latitude $b_{\mathrm{SG}}$ (illustrated by Fig. (6). The strongest excess of events corresponds to the band $\left|b_{\mathrm{SG}}\right|<15^{\circ}$ (region 1) while the region of the strongest deficit (region 2) is the Northern supergalactic hemisphere, $+15^{\circ}<b_{\mathrm{SG}}<+90^{\circ}$. For any of the space-based experiments, the optimal minimal energy of the sample is $E_{\min }=7 \cdot 10^{19} \mathrm{eV}$, and $\sim 30$ events with these energies are needed in the full-sky sample for $95 \%$ CL evidence/exclusion of the hypothesis.

\subsection{Results for the SHDM scenario}

Not surprisingly again, the regions 1 and 2 for the SHDM search are determined as cones of angle $\psi$ around the Galactic center and anti-center, respectively. The optimal parameters are given in Table 1 for different experiments.

\section{Conclusions}

In this work, we made quantitative predictions for the global anisotropy of the UHECR arrival directions expected in two distinct scenarios ("top-down" and "bottom-up") of the origin of the highest-energy cosmic rays.

Several refinements and improvements resulted in considerable changes in the 
Table 1. Optimized search parameters for the SHDM scenario. The columns give: (1), experiment name; (2), assumed SHDM plus extragalactic fit of the AGASA spectrum (see Sec. 3); (3)-(4), optimized search parameters: $E_{\min }-$ minimal energy of the sample, $\psi$ - angular radius of the cone around the Galactic Center (region 1) and Galactic anti-center (region 2); (5), number of events in the sample required for $95 \%$ CL evidence/exclusion of the hypothesis.

\begin{tabular}{ccccc}
\hline $\begin{array}{c}\text { Experiment } \\
(1)\end{array}$ & $\begin{array}{c}\text { Fit } \\
(2)\end{array}$ & $\begin{array}{c}E_{\min } \\
(3)\end{array}$ & $\begin{array}{c}\psi \\
(4)\end{array}$ & $\begin{array}{c}N \\
(5)\end{array}$ \\
\hline TUS & best & $5 \cdot 10^{19} \mathrm{eV}$ & $70^{\circ}$ & 138 \\
& $\min$ & $9 \cdot 10^{19} \mathrm{eV}$ & $70^{\circ}$ & 87 \\
\hline JEM-EUSO, & best & $4 \cdot 10^{19} \mathrm{eV}$ & $70^{\circ}$ & 265 \\
S-EUSO & min & $7 \cdot 10^{19} \mathrm{eV}$ & $60^{\circ}$ & 167 \\
\hline
\end{tabular}

predictions as compared to previous studies. In particular, the patterns in the distribution of arrival directions of cosmic rays from astrophysical sources are more pronounced than expected before. The superheavy dark matter scenarios consistent with current data predict less pronounced anisotropy.

We developed optimal observables for distinction (exclusion) of these two scenarios. The actual required observational time depends strongly on currently uncertain duty cycle, fluorescent yield and the actual spectrum. In any case, some of the scenarios will be tested even with the limited statistics of the first space-based UHECR detector, TUS, before (or at the time of) the planned launch of JEM/EUSO. However, only JEMEUSO and/or S-EUSO will be able to detect the SHDM component predicted by the "minimal" fit of the AGASA data consistent at 95\% CL with the spectrum and with photon limits.

To observe, at the $95 \% \mathrm{CL}$, the patterns correlated with cosmic large-scale structure, one needs $\sim 30$ events in the full-sky sample with energies $E>7 \cdot 10^{19} \mathrm{eV}$. If these patterns show up at high confidence with lower statistics, this might indicate either significant underestimation of the particle energies or a problem in theoretical understanding of the origin and/or propagation of ultra-high-energy cosmic particles. One may try to use these patterns as a rough but independent tool for the energy calibration, quite important for space-based detectors.

\section{Acknowledgments}

The authors are indebted to D. Gorbunov, G. Rubtsov and D. Semikoz for discussions. We acknowledge the use of online tools [28, 30, 33]. This work was supported in part by the grants RFBR 07-02-00820 (OK and ST) and NS-7293.2006.2 (government contract 02.445.11.7370, OK and ST) and by the Russian Science Support Foundation fellowship (ST). Simulations of the cosmic-ray propagation have been performed at the computer cluster of the Theoretical Division of INR RAS. 


\section{Appendix A. Construction of the density function of the sources}

\section{Appendix A.1. The 2MASS sample}

The most complete full-sky catalog of galaxies, the 2MASS XSC [28], does not contain distances to objects listed there (simply because their redshifts have not been measured in most cases). However, one may use the "photometric reshifts" 29] assuming that the galaxies are standard candles in the infrared. More precisely, one assumes that all galaxies have the same absolute magnitudes in $K$-band, $M_{K}=M_{\star}$ (this assumption works good enough in average for large samples of galaxies, see discussion in Ref. [29]). Following Refs. [29, 45], we assume $M_{\star}=-24.0$. One has [29]

$$
M_{K}=K_{\mathrm{corr}}-5 \log \frac{D_{L}(z)}{r_{0}}-k(z),
$$

where $K_{\text {corr }}$ is the apparent $K$-magnitude corrected for the Galactic extinction, $D_{L}(z)$ is the luminosity distance (for redshifts $z \lesssim 0.1$ which we are interested in, $D_{L}(z) \approx z / H_{0}$; we use the Hubble constant $\left.H_{0}=73 \mathrm{~km} \cdot \mathrm{s}^{-1} \cdot \mathrm{Mpc}^{-1}\right), r_{0}=10 \mathrm{pc}$ and $k(z)$ is the cosmic-reddening correction, $k(z) \approx-6 \log (1+z)$. The distance $d$ to a galaxy is then determined as

$$
d=\frac{5}{12}\left(1-\sqrt{1-\frac{24}{5} H_{0} r_{0} 10^{\left(K_{\mathrm{corr}}-M_{\star}\right) / 5}}\right) H_{0}^{-1} .
$$

We use the model of Ref. [33] for correcting the observed $K$-magnitude $K_{\text {obs }}$ for the Galactic extinction:

$$
K_{\text {corr }}=K_{\text {obs }}-0.367 E(B-V),
$$

where $E(B-V)$ is found for a given direction $(l, b)$ by making use of the code dust_getval available from the website [33].

According to Ref. [29], the 2MASS XSC is a complete catalog of galaxies with $|b| \geq 5^{\circ}$ and $K<13^{m}$. This corresponds to the photometric redshift $z \approx 0.066$ (that is, $d \approx 270 \mathrm{Mpc})$

\section{Appendix A.2. The LEDA sample and matching}

The photometric redshifts at low distances are not always reliable because of their relatively low precision (which affects the results more significantly for closeby objects). Moreover, the sample of nearby galaxies determined by photometric redshifts suffer from contamination by Galactic objects (though firm Galactic identifications are listed in a catalog available from the XSC website [28] and can easily be removed, one expects that a fraction of these objects is still present) and by galaxies for which the galactic extinction was determined poorly (e.g. due to molecular clouds in front of them). Therefore, one needs another source of data at small distances $d$.

The most complete optical full-sky catalog of galaxies is the LEDA database [30]. It contains information about radial velocities (known mostly for nearby galaxies). The completeness of the catalog has been studied in Ref. [31] where volume-limited complete 


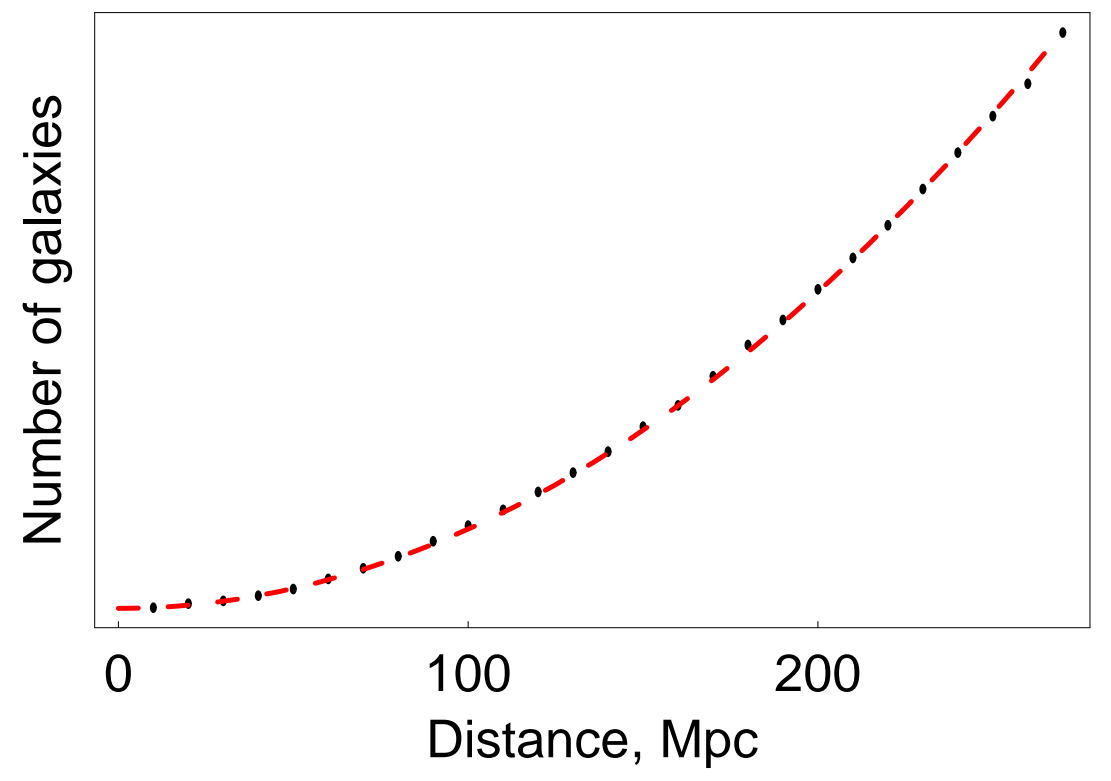

Figure A1. The number of galaxies in our sample in slices of $10 \mathrm{Mpc}$ in distance, as a function of distance. The dashed line represents the quadratic fit expected for a complete sample.

samples were determined. We use the sample of galaxies with known distances up to $50 \mathrm{Mpc}$, complete for absolute $B$-magnitudes $M_{B}<-19$ and $|b| \geq 15^{\circ}$. From this sample, we construct the source density function $n_{\mathrm{LEDA}}(l, b, d)$ and compare it to $n_{\mathrm{XSC}}(l, b, d)$ in the region $30 \mathrm{Mpc} \leq d \leq 50 \mathrm{Mpc}$ to determine the relative normalization of the two functions:

$$
n_{\mathrm{XSC}} \simeq 1.03 n_{\mathrm{LEDA}} .
$$

The normalization factor is close to unity which reflects the completeness of the both samples. For our study, we therefore use the function

$$
n(l, b, d)= \begin{cases}1.03 n_{\mathrm{LEDA}}(l, b, d), & d \leq 30 \mathrm{Mpc} \\ n_{\mathrm{XSC}}(l, b, d), & 30 \mathrm{Mpc}<d \leq 270 \mathrm{Mpc} .\end{cases}
$$

Though $n_{\mathrm{XSC}}$ is determined for $|b| \geq 5^{\circ}$, we have to limit ourselves to $|b| \geq 15^{\circ}$ because of the zone-of-avoidance cut in the LEDA sample.

When the $\mathrm{XSCz}$ catalog [46] (see also Ref. [27]) containing distances to many 2MASS galaxies will become available, the use of the separate database for nearby galaxies will be not necessary and it will be possible to include the region $5^{\circ} \leq|b|<15^{\circ}$ in the study.

The total number of galaxies in our sample is 212700. The completeness of our catalog is illustrated by Fig. A1. 
Global anisotropy of cosmic rays with space-based detectors

\section{References}

[1] Waxman E, Fisher K B and Piran T, The signature of a correlation between $>10^{19}-e V$ cosmic ray sources and large scale structure, 1997 Astrophys. J. 4831 arXiv:astro-ph/9604005; Evans N W, Ferrer F and Sarkar S, The anisotropy of the ultra-high energy cosmic rays, 2002 Astropart. Phys. 17319 arXiv:astro-ph/0103085]; Smialkowski A, Giller M and Michalak $\mathrm{W}$, Luminous infrared galaxies as possible sources of the UHE cosmic rays, 2002 J. Phys. G 281359 arXiv:astro-ph/0203337; Sigl G, Miniati F and Ensslin T A, Ultra-high energy cosmic ray probes of large scale structure and magnetic fields, 2004 Phys. Rev. D 70043007 arXiv:astro-ph/0401084; Cuoco A et al., The footprint of large scale cosmic structure on the ultra-high energy cosmic ray distribution, 2006 JCAP 0601009 arXiv:astro-ph/0510765.

[2] Greisen K, End To The Cosmic Ray Spectrum?, 1966 Phys. Rev. Lett. 16 748; Zatsepin G T and Kuzmin V A, Upper limit of the spectrum of cosmic rays, 1966 JETP Lett. 478 [Pisma Zh. Eksp. Teor. Fiz. 4 114]

[3] Berezinsky V, Kachelrieß M and Vilenkin A, Ultra-high energy cosmic rays without GZK cutoff, 1997 Phys. Rev. Lett. 794302 arXiv:astro-ph/9708217]; Kuzmin V A and Rubakov V A, Ultrahigh-energy cosmic rays: A window on postinflationary reheating epoch of the universe?, 1998 Phys. Atom. Nucl. 611028 [Yad. Fiz. 61 1122] arXiv:astro-ph/9709187]

[4] Bhattacharjee P and Sigl G, Origin and propagation of extremely high energy cosmic rays, 2000 Phys. Rept. 327109 arXiv:astro-ph/9811011 Kachelrieß M, Status of particle physics solutions to the UHECR puzzle, 2004 Comptes Rendus Physique 5441

[5] Dubovsky S L and Tinyakov P G, Galactic anisotropy as signature of CDM-related ultra-high energy cosmic rays, 1998 JETP Lett. 68107 arXiv:hep-ph/9802382

[6] Berezinsky V and Mikhailov A, Anisotropy of ultra high energy cosmic rays in the dark matter halo models, 1999 Phys. Lett. B 449, 237 [arXiv:astro-ph/9810277]; Medina-Tanco G A and Watson A A, Dark matter halos and the anisotropy of ultra-high energy cosmic rays, 1999 Astropart. Phys. 1225 arXiv:astro-ph/9903182

[7] Aloisio R, Berezinsky V and Kachelrieß M, Ultra high energy cosmic rays spectra in top-down models, 2004 Nucl. Phys. Proc. Suppl. 136319 arXiv:astro-ph/0409222

[8] Aloisio R, Berezinsky V and Kachelrieß M, On the status of superheavy dark matter, 2006 Phys. Rev. D 74023516 arXiv:astro-ph/0604311.

[9] Takeda M et al., Energy determination in the Akeno Giant Air Shower Array experiment, 2003 Astropart. Phys. 19447 arXiv:astro-ph/0209422

[10] Abbasi R et al., (HiRes Collaboration), Observation of the GZK cutoff by the HiRes experiment, arXiv:astro-ph/0703099.

[11] Roth M et al. (Pierre Auger Collaboration), Measurement of the UHECR energy spectrum using data from the Surface Detector of the Pierre Auger Observatory, Proc. 30th ICRC (2007), arXiv:0706.2096 [astro-ph].

[12] Engel R et al. (Pierre Auger Collaboration), Test of hadronic interaction models with data from the Pierre Auger Observatory, Proc. 30th ICRC (2007). arXiv:0706.1921 [astro-ph].

[13] Rubtsov G I et al., Upper limit on the ultra-high-energy photon flux from AGASA and Yakutsk data, 2006 Phys. Rev. D 73063009

[14] Glushkov A V et al., Constraining the fraction of primary gamma rays at ultra-high energies from the muon data of the Yakutsk extensive-air-shower array, 2007 JETP Lett. 85131 arXiv:astro-ph/0701245

[15] Healy M D et al. (Pierre Auger Collaboration), Search for Ultra-High Energy Photons with the Pierre Auger Observatory, Proc. 30th ICRC (2007), arXiv:0710.0025 [astro-ph].

[16] Risse M and Homola P, Search for ultra-high energy photons using air showers, 2007 Mod. Phys. Lett. A 22749 arXiv:astro-ph/0702632

[17] Abraham J et al. [Pierre Auger Collaboration], 2007, Correlation of the highest energy cosmic rays with nearby extragalactic objects, Science 318938 arXiv:0711.2256. 
[18] Gorbunov D et al., 2007, Comment on 'Correlation of the Highest-Energy Cosmic Rays with Nearby Extragalactic Objects', arXiv:0711.4060.

[19] Abrashkin V I et al., The TUS space fluorescence detector for study of UHECR and other phenomena of variable fluorescence light in the atmosphere, 2006 Adv. Space Research 37 1876; Abrashkin V et al., Status of the TUS space detector preparation for UHECR study, Proc. 30th ICRC (2007).

[20] Ebisuzaki T et al., The JEM-EUSO Mission, Proc. 30th ICRC (2007).

[21] Santangelo A et al., S-EUSO: a proposal for a space-based observatory of Ultra-high-Energy cosmic particles, submitted to the ESA Cosmic Vision 2015-2025 Program (2007).

[22] Mattox J R et al., The Likelihood Analysis of EGRET Data, 1996 Astrophys. J. 461 396; Tompkins $\mathrm{W}$, Applications of likelihood analysis in gamma-ray astrophysics, arXiv:astro-ph/0202141; Gorbunov D S et al., Identification of extragalactic sources of the highest energy EGRET photons by correlation analysis, 2005 Mon. Not. Roy. Astron. Soc. Lett. 362 L30 arXiv:astro-ph/0505597

[23] Sommers P, Cosmic Ray Anisotropy Analysis with a Full-Sky Observatory, 2001 Astropart. Phys. 14271 arXiv:astro-ph/0004016

[24] Torres D F and Anchordoqui L A, Astrophysical origins of ultrahigh energy cosmic rays, 2004 Rept. Prog. Phys. 671663 arXiv:astro-ph/0402371]; Gorbunov D and Troitsky S, A comparative study of correlations between arrival directions of ultra-high-energy cosmic rays and positions of their potential astrophysical sources, 2005 Astrop. Phys. 23175 arXiv:astro-ph/0410741

[25] Kalashev O E, Kuzmin V A and Semikoz D V, Ultra high energy cosmic rays propagation in the galaxy and anisotropy, 2001 Mod. Phys. Lett. A 162505 arXiv:astro-ph/0006349; Kalashev O E, Kuzmin V A and Semikoz D V, Top-down models and extremely high energy cosmic rays, arXiv:astro-ph/9911035.

[26] Saunders W et al., The PSCz Catalogue, 2000 Mon. Not. Roy. Astron. Soc. 31755 arXiv:astro-ph/0001117

[27] Huchra J et al., The 2MASS redshift survey, http://cfa-www.harvard.edu/`huchra/2mass/

[28] Jarrett $\mathrm{T} \quad \mathrm{H}$ et al., 2MASS Extended Source Catalog: Overview and Algorithms, 2000 Astron. J. 1192498 arXiv:astro-ph/0004318; http://irsa.ipac.caltech.edu/cgi-bin/Gator/nph-dd?catalog=fp_xsc

[29] Jarrett T, Large Scale Structure in the Local Universe: The 2MASS Galaxy Catalog, arXiv:astro-ph/0405069

[30] Paturel G et al., HYPERLEDA. I. Identification and designation of galaxies, 2003 Astron. Astrophys. 412 45; http://leda.univ-lyon1.fr

[31] Courtois $\mathrm{H}$ et al., The LEDA galaxy distribution: I. Maps of the Local Universe, 2004 Astron. Astrophys. 42327 arXiv:astro-ph/0403545

[32] Jarrett T H et al., 2MASS Galaxy Colors: Hercules Cluster, 1998 Bull. Amer. Astron. Soc. 30 901; Kochanek C S et al., Clusters of galaxies in the local universe, 2003 Astrophys. J. 585, 161 arXiv:astro-ph/0208168

[33] Schlegel D J, Finkbeiner D P and Davis M, Maps of Dust IR Emission for Use in Estimation of Reddening and CMBR Foregrounds, 1998 Astrophys. J. 500525 arXiv:astro-ph/9710327; http://astro.berkeley.edu/dust/index.html

[34] Gelmini G, Kalashev O and Semikoz D V, GZK photons in the minimal ultra high energy cosmic rays model, arXiv:astro-ph/0702464.

[35] Arisaka K et al., Composition of UHECR and the Pierre Auger Observatory Spectrum, arXiv:0709.3390 [astro-ph].

[36] Harari D, Mollerach S and Roulet E, On the ultra-high energy cosmic ray horizon, 2006 JCAP 0611012 arXiv:astro-ph/0609294

[37] Dolag K et al., Mapping deflections of Ultra-High Energy Cosmic Rays in Constrained Simulations of Extragalactic Magnetic Fields, 2004 JETP Lett. 79583 arXiv:astro-ph/0310902; Dolag K et al., Constrained simulations of the magnetic field in the local universe and the propagation of 
UHECRs, 2005 JCAP 0501009 arXiv:astro-ph/0410419.

[38] Sigl G, Miniati F and Ensslin T A, Signatures of magnetized large scale structure in ultrahigh energy cosmic rays, arXiv:astro-ph/0309695; Sigl G, Miniati F and Ensslin T A, Cosmic magnetic fields and their influence on ultra-high energy cosmic ray propagation, 2004 Nucl. Phys. Proc. Suppl. 136224 arXiv:astro-ph/0409098].

[39] Globus N, Allard D and Parizot E, Propagation of high-energy cosmic rays in extragalactic turbulent magnetic fields: resulting energy spectrum and composition, arXiv:0709.1541 [astro-ph].

[40] Tinyakov P G and Tkachev I I, Tracing protons through the galactic magnetic field: A clue for charge composition of ultrahigh-energy cosmic rays, 2002 Astropart. Phys. 18165 arXiv:astro-ph/0111305; Prouza M and Smida R, The Galactic magnetic field and propagation of ultra-high energy cosmic rays, 2003 Astron. Astrophys. 4101 arXiv:astro-ph/0307165; Troitsky S V, Magnetic deflections and possible sources of clustered ultra-high-energy cosmic rays, 2006 Astropart. Phys. 26325 arXiv:astro-ph/0505262; Kachelriess M, Serpico P D and Teshima M, The Galactic magnetic field as spectrograph for ultra-high energy cosmic rays, 2006 Astropart. Phys. 26378 arXiv:astro-ph/0510444].

[41] Sarkar S and Toldra R, The high energy cosmic ray spectrum from massive particle decay, 2002 Nucl. Phys. B 621495 arXiv:hep-ph/0108098; Barbot C and Drees M, Production of ultraenergetic cosmic rays through the decay of super-heavy X particles, 2002 Phys. Lett. B 533107 arXiv:hep-ph/0202072; Barbot C and Drees M, Detailed analysis of the decay spectrum of a super-heavy X particle, 2003 Astropart. Phys. 205 arXiv:hep-ph/0211406

[42] Chung D J H et al., Isocurvature constraints on gravitationally produced superheavy dark matter, 2005 Phys. Rev. D 72023511 arXiv:astro-ph/0411468

[43] Navarro J F, Frenk C S and White S D M, The Structure of Cold Dark Matter Halos, 1996 Astrophys. J. 462563 arXiv:astro-ph/9508025

[44] Gorbunov D S et al., Estimate of the correlation signal between cosmic rays and BL Lacs in future data, 2006 JCAP 0601025 arXiv:astro-ph/0508329

[45] Cole S et al. (The 2dFGRS Collaboration), The 2dF Galaxy Redshift Survey: Near Infrared Galaxy Luminosity Functions, 2001 Mon. Not. Roy. Astron. Soc. 326255 arXiv:astro-ph/0012429; Kochanek C S et al., The K-Band Galaxy Luminosity Function, 2001 Astrophys. J. 560566 arXiv:astro-ph/0011456

[46] Jarrett T, 2MASS Galaxy Redshift Catalog (XSCz), http://spider.ipac.caltech.edu/staff/jarrett/XSCz/index.html 